
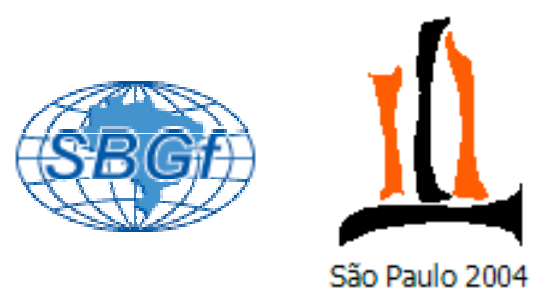

\title{
Anomalias Geoidais e a Estrutura da Litosfera no Cráton São Francisco Meridional
}

Luiz Gabriel S. de Oliveira, Maria Sílvia C. Barbosa, Issamu Endo - DEGEO/EM/UFOP

Denizar Blitzkow - EP/USP

\section{Copyright 2004, SBGf - Sociedade Brasileira de Geofísica}

Este texto foi preparado para a apresentação no I Simpósio de Geofísica da Sociedade Brasileira de Geofísica, São Paulo, 26-28 de setembro de 2004. Seu conteúdo foi revisado pela Comissão Tecno-científica do I SR-SBGf mas não necessariamente representa a opinião da SBGf ou de seus associados. E proibida a reprodução total ou parcial deste material para propósitos comerciais sem prévia autorização da SBGt.

\section{Resumo}

Um modelo preliminar de densidades para a litosfera na região do Cráton São Francisco Meridional e adjacências foi determinado com base em anomalias geoidais obtidas do modelo geopotencial GGM01C, pertencente a missão espacial GRACE. A litosfera na região do Cráton São Francisco possui cerca de $150 \mathrm{~km}$ de espessura. Os parâmetros reológicos, módulo de rigidez e espessura elástica foram calculados em $2.27 \times 10^{24} \mathrm{~N} . \mathrm{m}$ e $70 \mathrm{~km}$, respectivamente, admitindo-se um modelo de compensação isostática flexural.

\section{Introdução}

O geóide, definido como a superfície equipotencial do campo de gravidade terrestre que mais se aproxima do nível médio não perturbado dos mares (Featherstone 1997), pode fornecer informações relevantes sobre a estrutura interna da Terra (Chapman 1979).

Diferentemente de levantamentos gravimétricos terrestres, as anomalias geoidais permitem lançar hipóteses sobre as distribuições de densidade ocorrentes no manto terrestre, pelo fato de trabalharem com os comprimentos de onda mais longos do campo de gravidade (Turcotte \& McAdoo 1979, Marquart \& Lelgemann 1992).

Neste contexto, este trabalho tem como objetivo apresentar um modelo preliminar para a litosfera na região do Cráton São Francisco Meridional e adjacências, além de determinar alguns de seus parâmetros reológicos. As anomalias geoidais aqui utilizadas foram determinadas com base em um modelo geopotencial fornecido a partir de dados provenientes da missão espacial GRACE (Rummel et al. 2002).

\section{Metodologia/ Problema Investigado}

A determinação das anomalias geoidais $(N)$ para a área de estudo, localizada num perfil compreendido entre os meridianos $48^{\circ} \mathrm{W}$ e $42^{\circ} \mathrm{W}$ e o paralelo $19^{\circ} \mathrm{S}$ (Figura 1 ), foi efetuada com base nos coeficientes do modelo geopotencial GGM01C, de grau e ordem 200, obtido a partir de informações (rastreio, aceleração dos satélites e altitude) colhidas durante 111 dias da missão espacial GRACE, que foram agregadas posteriormente a dados gravimétricos terrestres e de altimetria por satélite.
Os valores de $N$ foram calculados com base nas equações apresentadas por Gemael (2002), onde o potencial perturbador $(T)$ foi determinado pela subtração do potencial gravitacional teórico do potencial gravitacional obtido pelos coeficientes do modelo geopotencial GGM01C. Por meio da equação de Bruns

$$
N=T / \gamma
$$

onde $\gamma$ é a gravidade normal, os valores de $N$ ao longo do perfil foram determinados. O elipsóide de referência adotado foi o GRS80. Os procedimentos computacionais seguidos nesta etapa foram os apresentados por Smith (1998)

As anomalias geoidais determinadas a partir do modelo geopotencial GGM01C foram filtradas através da técnica detrending (Featherstone 1997), que retirou a influência de fontes associadas a profundidades maiores que $300 \mathrm{~km}$.

Por último, as anomalias geoidais filtradas foram corrigidas do efeito isostático (geóide residual), com o intuito de prover informações acerca da distribuição de densidades no manto litosférico (Rummel et al. 2002). Esta etapa seguiu os procedimentos teóricos contidos em Haxby \& Turcotte (1978), com espessura crustal de $35 \mathrm{~km}$ (Molina \& Ussami 1999) e dados topográficos tomados do modelo digital de elevação ETOPO5 (Figura 2).

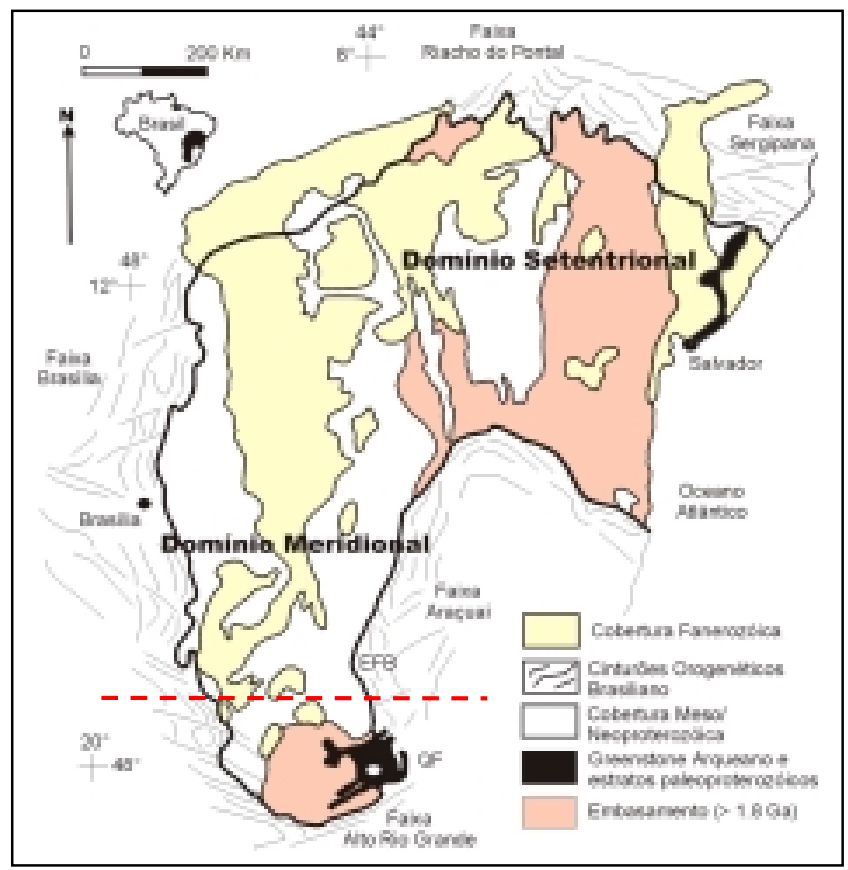

Figura 1 - Limites do Cráton São Francisco e localização (em tracejado) do perfil de anomalias geoidais (modificado de Alkmim et al. 1993). 


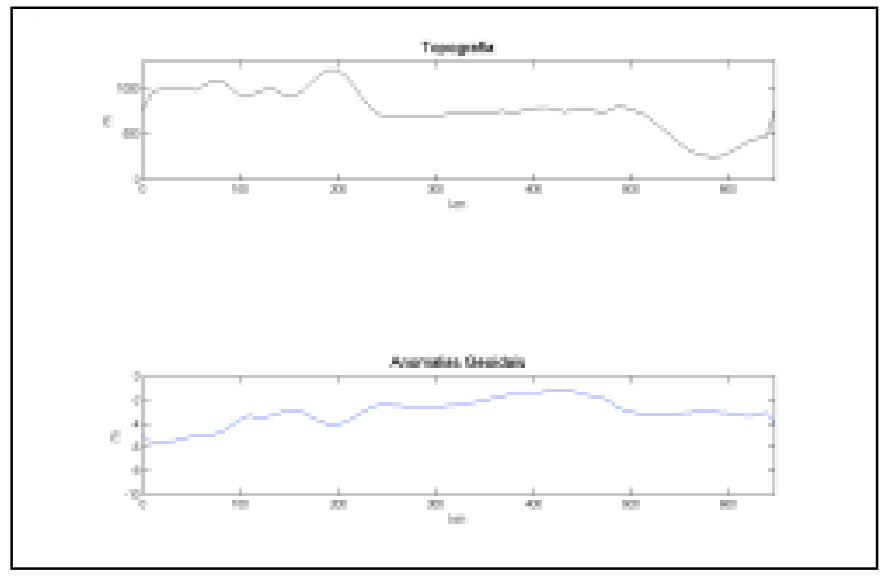

Figura 2 - Topografia e anomalias geoidais residuais modeladas neste estudo.

\section{Resultados}

Para o cálculo das anomalias geoidais teóricas referentes ao modelo litosférico, foi utilizada a equação de Parker (1972), moficada segundo as relações propostas entre anomalias gravimétrica e geoidal, no domínio da freqüência, mostradas por Chapman (1979). Assim, a equação

$$
N(k)=\frac{-2 \pi G}{g} \Delta \rho e^{-k z} \sum_{n=1}^{\infty} \frac{|k|^{n-2}}{n !} s^{n}(k)
$$

fornece a anomalia geoidal relativa a uma interface com contraste de densidade $\Delta \rho$ e geometria $s(k)$ localizada numa profundidade $z$. A variável $G$ refere-se à constante universal da gravitação, $g$ à aceleração da gravidade e $k$ representa 0 número de onda da freqüência $(k=2 \pi / \lambda)$, onde $\lambda$ é o comprimento de onda da interface.

O modelo aqui apresentado é constituído por 4 camadas: crosta superior $\left(\rho=2800 \mathrm{~kg} / \mathrm{m}^{3}\right)$, crosta inferior $(\rho=3100$ $\left.\mathrm{kg} / \mathrm{m}^{3}\right)$, manto litosférico $\left(\rho=3400 \mathrm{~kg} / \mathrm{m}^{3}\right)$ e manto astenosférico $\left(\rho=3210 \mathrm{~kg} / \mathrm{m}^{3}\right)$. Os valores de densidade foram compilados de Blitzkow et al. (1979), Marquart \& Lelgemann (1992) e Molina \& Ussami (1999).

A interface crosta-manto foi obtida com base na hipótese de compensação flexural, onde a deflexão $w$ causada por uma carga topográfica $h$ é obtida por (Turcotte \& schubert 2002)

$$
w=\frac{h}{\left[\left(\frac{\rho_{m}}{\rho_{c}}\right)-1+\left(\frac{D}{\rho_{m} g}\right)\left(\frac{2 \pi}{\lambda}\right)\right]}
$$

sendo $D$ o módulo de rigidez flexural, $g$ a aceleração da gravidade. A partir de $D$, é possível calcular a espessura elástica $(T e)$ pela relação

$$
D=\frac{E T e^{3}}{12\left(1-v^{2}\right)}
$$

onde $E$ é o módulo de Young $\left(0.75 \times 10^{11} \mathrm{~Pa}\right)$ e $v$ o coeficiente de Poisson (0.25).

As equações 2, 3 e 4 serviram de base para uma rotina computacional de modelagem 2D desenvolvida com auxilio do software MATLAB. Foram testados vários valores de $T e$, sendo que o melhor ajuste foi atingido com $T e=70 \mathrm{~km}$. A geometria do limite litosfera-astenosfera foi alterada, contribuindo para o melhor ajuste entre os valores calculados e observados.

\section{Discussão e Conclusões}

Este trabalho apresentou uma metodologia de estudo preliminar da estrutura da litosfera terrestre, utilizando-se modelos geopotenciais.

O modelo 2D (Figura 3) apresenta uma possível distribuição de densidades para a região do Cráton São Francisco Meridional e adjacências. Alguns pontos devem ser destacados:

a) a variação de espessura crustal é nítida, possuindo valores entre $40-42 \mathrm{~km}$ na região do Cráton São Francisco e no intervalo de $36-38 \mathrm{~km}$ nas faixas de dobramentos. Estes resultados corroboram com os alcançados por Blitzkow et al. (1979) e com os dados de refração sísmica obtidos por Giese \& Schutte (1980) na região;

b) a espessura da litosfera na região do Cráton São Francisco Meridional é de cerca de $150 \mathrm{~km}$, enquanto que nas faixas de dobramentos situa-se entre 100$110 \mathrm{~km}$. Este resultado se encaixa nos valores de espessura termal calculados por Artemieva (1998);

c) a discrepância entre os valores de $N$ calculados e observados, tanto na Faixa Brasília como na Faixa Araçuaí, pode estar relacionada a fontes de menor densidade presentes no manto litosférico ou na base da crosta inferior;

d) o valor de $T e$ determinado $(70 \mathrm{~km})$ é maior do que o calculado por Ortu (1990), através do uso da função de transferência entre a topografia e as anomalias de gravidade (15-30km). Porém, se aproxima do intervalo de valores $(76-89 \mathrm{~km})$ determinado por Mantovani et al. (2001), obtido pela correlação empírica de anomalias de maré gravimétrica e Te. Uma possível justificativa para este fato é que o uso de anomalias geoidais, que estão associadas aos comprimentos de onda mais longos do campo gravitacional, permite trabalhar com a distribuição de densidades em partes mais profundas do manto (litosfera-astenosfera), que podem ser associadas ao estado termal da litosfera, o que afeta diretamente os valores de Te. 


\section{Agradecimentos}

Os autores agradecem a NASA pelo uso do modelo geopotencial GGM01C.

\section{Referências}

Alkmim F. F., Brito Neves B. B. \& Alves J. C., 1993. Arcabouço tectônico do Cráton São Francisco: uma revisão. In: J. M. L. Dominguez \& A. Misi. (eds.), O Cráton do São Francisco. Salvador, SGM/SBG-BA, 4562.

Artemieva, I. M., 1998. Thermal state of the archean and proterozoic lithosphere: global results for thermal thickness, subcrustal temperatures and composition. Proceedings 14th. International Conference on Basement Tectonics, Ouro Preto (MG), Brazil: 102-103.

Blitzkow, D., Gasparini, P., de Sá, N. C., Mantovani, M. S. M., 1979. Crustal structure of southeastern Minas Gerais, Brazil, deduced from gravity measurements. Rev. Bras. Geoc., 9: 39-43.

Chapman, M., 1979. Techniques for interpretation of geoid anomalies. J. Geophys. Res., 84(B8): 3793-3802.

Featherstone, W. E., 1997. On the use of geoid in Geophysics: a case study over the northwest shelf of Australia. Explor. Geophys., 28(1): 52-57.

Gemael, C., 2002. Introdução à Geodésia Física. 2nd ed., Editora UFPR, Curitiba, Brasil.

Giese, P. \& Schutte, K. G. 1980. Resultados das medidas de sísmica de refração a leste da Serra do Espinhaço, MG, Brasil. Relatório não-publicado, Instituto de Geofísica, Universidade de Berlin.

Haxby, W. F. \& Turcotte, D. L., 1978. On isostatic geoid anomalies. J. Geophys. Res., 83(B11): 5473-5478.

Mantovani, M. S. M., de Freitas, S. R. C. \& Shukowsky, W., 2001. Tidal gravity anomalies as a tool to measure rheological properties of continental lithosphere: application to the South American Plate. J. South Americ. Earth Sci., 14(1): 1-14.

Marquart, G. \& Lelgemann, D., 1992. On the interpretation of geoid anomalies in Europe with special regard to the EGT profiles. Tectonophys., 207: 25-42.

Molina, E. C. \& Ussami, N., 1999. The geoid in southeastern Brazil and adjacent regions: new constrains on density distribution and thermal state of the lithosphere. J. Geodyn., 28: 357-374.

Ortu, J. C., 1990. Modelagem tectono-geofísica da porção sul da bacia do São Francisco, Minas Gerais. Dissertação de mestrado, Departamento de Geologia da Universidade Federal de Ouro Preto, Minas Gerais.
Parker, R. L., 1972. The rapid calculation of potential anomalies. Geophys. J. R. Astron. Soc., 31: 447-455.

Rummel, R., Balmino, G., Johanessen, J., Visser, P. \& Woodworth, P., 2002. Dedicated gravity field missions principles and aims. J. Geodyn., 33: 3-20.

Smith, D. A., 1998. There is no such thing as "The" EGM96 geoid: subtle points on the use of a global geopotential model. IGeS Bull., 8: 17-28.

Turcotte, D. L. \& McAdoo, D. C., 1979. Geoid anomalies and thickness of the lithosphere. J. Geophys. Res., 84(B5): 2831-2837.

Turcotte D. L. \& Schubert G., 2002. Geodynamics. 2nd ed., John Wiley \& Sons, Nova York, USA. 

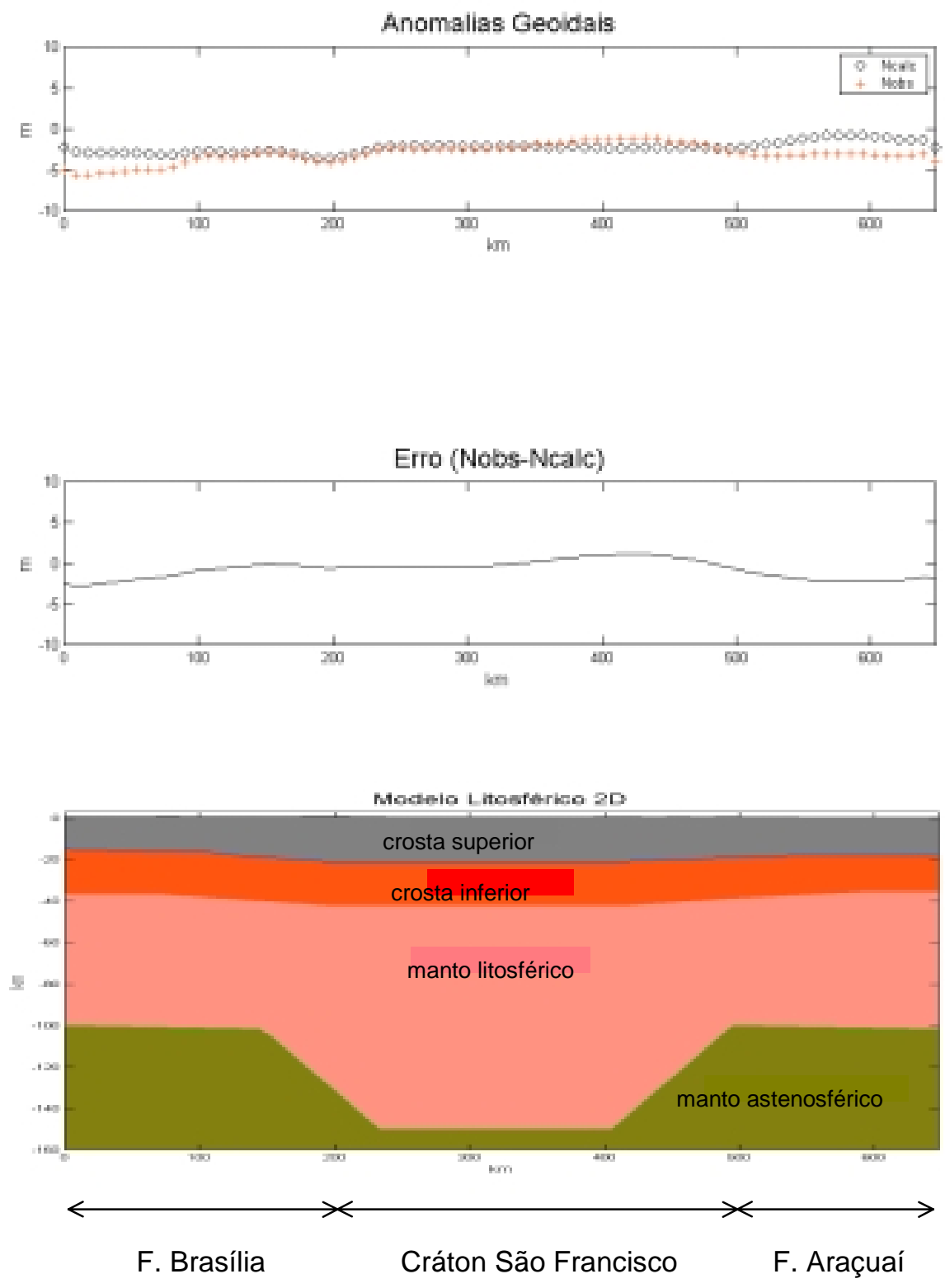

Figura 3 - Modelo Litosférico 2D na região do cráton São Francisco Meridional e adjacências, baseado nos coeficientes do modelo geopotencial GGM01C. 